\title{
A Micropolar Material Model for Turbulent SPH Fluids
}

\author{
Jan Bender \\ RWTH Aachen University \\ bender@cs.rwth-aachen.de \\ Tassilo Kugelstadt \\ RWTH Aachen University \\ kugelstadt@cs.rwth-aachen.de
}

\author{
Dan Koschier \\ RWTH Aachen University \\ koschier@cs.rwth-aachen.de \\ Marcel Weiler \\ TU Darmstadt \\ weiler@gsc.tu-darmstadt.de
}
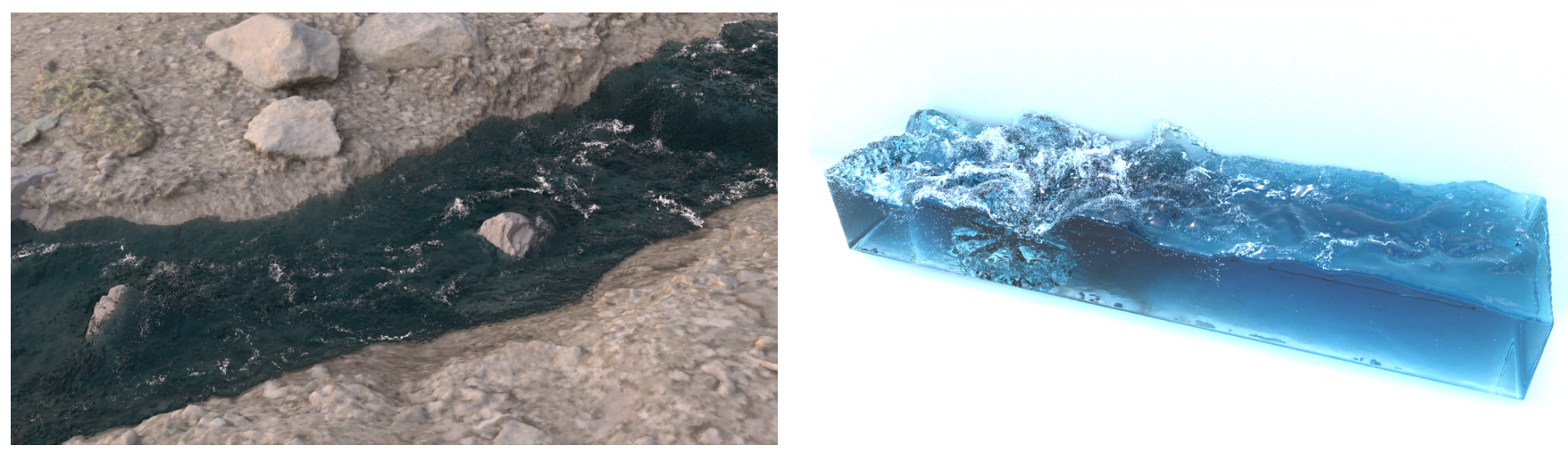

Figure 1: Our novel micropolar fluid model allows the simulation of complex turbulent fluids with static and dynamic boundaries. Left: A turbulent river with 12 million fluid particles flows through a complex static boundary. Right: One million fluid particles interact with a fast rotating propeller.

\begin{abstract}
In this paper we introduce a novel micropolar material model for the simulation of turbulent inviscid fluids. The governing equations are solved by using the concept of Smoothed Particle Hydrodynamics (SPH). As already investigated in previous works, SPH fluid simulations suffer from numerical diffusion which leads to a lower vorticity, a loss in turbulent details and finally in less realistic results. To solve this problem we propose a micropolar fluid model. The micropolar fluid model is a generalization of the classical NavierStokes equations, which are typically used in computer graphics to simulate fluids. In contrast to the classical Navier-Stokes model, micropolar fluids have a microstructure and therefore consider the rotational motion of fluid particles. In addition to the linear velocity field these fluids also have a field of microrotation which represents existing vortices and provides a source for new ones. However, classical micropolar materials are viscous and the translational and the rotational motion are coupled in a dissipative way. Since our goal is to simulate turbulent fluids, we introduce a novel modified micropolar material for inviscid fluids with a non-dissipative coupling. Our model can generate realistic turbulences, is linear and angular momentum conserving, can be easily integrated in existing SPH simulation methods and its computational overhead is negligible.
\end{abstract}

SCA '17, Los Angeles, CA, USA

(C) 2017 ACM. This is the author's version of the work. It is posted here for your personal use. Not for redistribution. The definitive Version of Record was published in Proceedings of SCA '17, fuly 28-30, 2017, https://doi.org/10.1145/3099564.3099578.

\section{CCS CONCEPTS}

- Computing methodologies $\rightarrow$ Physical simulation;

\section{KEYWORDS}

Smoothed Particle Hydrodynamics, micropolar fluids, turbulence, incompressible fluids

\section{ACM Reference format:}

Jan Bender, Dan Koschier, Tassilo Kugelstadt, and Marcel Weiler. 2017. A Micropolar Material Model for Turbulent SPH Fluids. In Proceedings of SCA '17, Los Angeles, CA, USA, July 28-30, 2017, 8 pages.

DOI: $10.1145 / 3099564.3099578$

\section{INTRODUCTION}

Lagrangian fluid simulation methods based on the Smoothed Particle Hydrodynamics (SPH) approach have been investigated for several years in the field of computer graphics. An important application area is the generation of special effects in movies where a main goal is the realistic simulation of incompressible fluids in turbulent scenarios. In recent years several methods to enforce incompressibility have been introduced. However, the generation of realistic turbulent flows in Lagrangian fluid simulations is still a challenging problem.

In SPH fluid simulations turbulent details quickly get lost due to numerical diffusion [de Goes et al. 2015] or due to a coarse sampling of the velocity field [Cornelis et al. 2014; Ihmsen et al. 2014b] which negatively influences the visual liveliness of the flow. In the field of Eulerian smoke simulations the concept of vorticity confinement 
was introduced to the graphics community by Fedkiw et al. [2001] to counteract numerical dissipation and to amplify existing vorticity. Later, this concept was adapted in SPH fluid simulations to obtain a more detailed motion of bubbles [Hong et al. 2008] and to counteract numerical damping [Macklin and Müller 2013]. However, these $\mathrm{SPH}$ variants of vorticity confinement are not momentum conserving and only existing vortices can be amplified. Lagrangian vortex methods, which are based on a vorticity representation of the Navier-Stokes equations, are an alternative approach to simulate turbulent fluids. They maintain a divergence-free velocity field and have no numerical dissipation. However, the handling of non-rigid obstacles and free surface flows is difficult using these methods.

In this paper we introduce an SPH simulation method for incompressible turbulent fluids to solve the above-mentioned problems. Our method is based on a novel micropolar material model for fluids. The theory of micropolar fluids is well-established in the field of mechanical engineering [Eringen 2001; Lukaszewicz 1999]. A micropolar fluid model is a generalization of the classical NavierStokes model for fluids. In contrast to the classical fluid model, micropolar fluids have a microstructure, i.e. they consist of rigid, spherical microelements. This results in a non-symmetric stress tensor. Therefore, the governing equations of the micropolar fluid model consider the rotational motion of the fluid particles. Or more specifically each particle has an angular velocity which defines a field of microrotation. In this field, vortices can exist independently of the linear velocity field. Moreover, this field provides a source for new vortices [Eringen 2001].

In our work we are interested in the simulation of inviscid turbulent fluids. However, the classical micropolar material is intended for viscous flow and couples the translational and the rotational motion in a dissipative way. Therefore, we introduce a modified micropolar material model which is specially tailored for the simulation of inviscid turbulent fluids. Our micropolar fluid model is linear and angular momentum conserving. Additionally, it is easy to implement, turbulences can be controlled with a single parameter and it can be combined with any existing SPH pressure solver without additional effort. Furthermore, the computational overhead compared to a classical SPH simulation is negligible. Finally, to the best of our knowledge, we are the first to introduce a micropolar fluid model in the field of computer graphics and the first to solve the governing equations for a micropolar model using SPH.

Our method is able to perform realistic simulations of complex turbulent fluid scenarios with several million particles as demonstrated in Figure 1. Moreover, it provides a more realistic visual liveliness of the flow than classical SPH fluids as we show in different comparisons in Section 6.

\section{RELATED WORK}

In the field of computer graphics, the simulation of turbulent fluids has been a topic of active research for nearly two decades. Moreover, SPH based methods for the simulation of free surface flow of incompressible fluids have gained popularity over the last years. In this section, we will discuss related work in the fields of SPH-based simulation, turbulent fluid simulation and micropolar material models and organized the discussion respectively. For a general survey on SPH based fluid simulations we would like to refer the reader to the state-of-the-art report of Ihmsen et al. [2014b].

SPH for Incompressible Fluids In recent years, a lot of attention has been paid to the development of implicit pressure solvers in order to efficiently enforce incompressibility in SPH based fluid simulations. Existing approaches can be mainly categorized into methods that iteratively project particle positions onto an uncompressed state and pressure projection methods that use either the divergence of the velocity field or the compression on position level as source term (cf. [Ihmsen et al. 2014b]). Solenthaler and Pajarola [2009] and Macklin and Müller [2013] proposed predictivecorrective approaches that iteratively project particle positions onto an uncompressed state. Approaches following the strategy of pressure projections were proposed by Ihmsen et al. [2014a] using the compression as source term and Premože et al. [2003] using the velocity divergence as source term. Recently, an approach enforcing both zero compression and a divergence-free velocity field was proposed by Bender and Koschier [2017]. In this work, we adopted their approach due to its computational efficiency and stability properties. However, our micropolar model is independent of the pressure solver and can therefore be easily used in combination with other solvers.

A known problem of SPH approaches is that they introduce numerical damping which is particularly evident in case of coarse discretizations (cf. [de Goes et al. 2015; Ihmsen et al. 2014b]). From a physical point of view, this leads to an undesired dissipation where high-frequency features are smoothed out as discussed by Ihmsen et al. [2014b]. It also reduces vorticity and prevents turbulences from forming (cf. [Cornelis et al. 2014]). Existing approaches that recover or amplify turbulent details can be mainly categorized into vorticity confinement, Lagrangian vortex methods and fluid up-sampling which will be discussed in the following.

Vorticity Confinement Fedkiw et al. [2001] introduced vorticity confinement to the computer graphics community to solve the issue of numerical dissipation in Eulerian simulations of smoke. The core idea is to identify existing vortices and to subsequently counteract dissipation of the rotational flow by amplification. Lentine et al. [2011] extended this work to achieve energy conservation and momentum conserving vorticity confinement forces. Jang et al. [2010] used a multilevel vorticity confinement approach to achieve turbulences in Eulerian water simulations. Vorticity confinement was also used in hybrid simulations, in which the fluids were simulated as SPH particles and where an additional grid was used for vorticity confinement. For instance Zhu et al. [2010] used a hybrid method and vorticity confinement to simulate fine scale vorticial details around moving rigid objects in SPH smoke simulations. Hong et al. [2008] used vorticity confinement in a simulation of bubbles in water. The water was simulated on a grid and the bubbles as SPH particles. They used vorticity confinement on the particles to achieve a more detailed motion. Also in purely particle based approaches vorticity confinement was used. Macklin and Müller [2013] determined the existing vorticity per particle using SPH and computed forces which accelerate the particles to amplify the rotational motion. 
Our experiments show that using vorticity confinement directly on SPH particles is a simple way to counteract numerical dissipation, but it cannot create additional turbulent details such as additional vortices. Moreover, the previously proposed vorticity confinement in SPH is not momentum conserving and it also violates energy conservation when the parameter is chosen too high, so that it adds more energy than numerical dissipation has removed. Unfortunately, the energy and momentum conserving Eulerian approach of Lentine et al. [2011] can not directly be applied to SPH simulations. In contrast, our method is able to create additional turbulent detail resulting in a feature-rich flow without the need for data structures like Eulerian grids and is linear and angular momentum conserving.

Lagrangian Vortex Methods Another approach is to simulate turbulent fluids by using the vorticity representation of the NavierStokes equations. This method has several advantages. It allows a compact representation of rotational flow features such as eddies, it automatically guarantees divergence-free velocity fields and it does not suffer from numerical dissipation. The vorticity field can be discretized using particles [Park and Kim 2005; Selle et al. 2005], curves/filaments [Angelidis and Neyret 2005; Weißmann and Pinkall 2009], or surfaces/sheets [Pfaff et al. 2012; Weißmann and Pinkall 2010]. Further, Zhang et al. [2015] proposed a hybrid vorticity and velocity based advection scheme to restore the missing vorticity in Eulerian solvers.

However, the vortex representation also has several disadvantages. It is difficult to handle boundaries such as non-rigid obstacles and free surface flows. Therefore, a hybrid Eulerian and vorticity method was proposed by Golas et al. [2012]. They used Lagrangian vortex elements in the interior of the fluid and boundaries were treated on an Eulerian grid. A further difficulty is that the velocity field has to be recovered by solving Biot-Savart integrals or a vector valued Poisson equation [Zhang and Bridson 2014].

The vorticity based models are related to our method because of the close relationship between vorticity and microrotations. However, in our approach these quantities are coupled by the (angular) momentum equations, i.e. we do not have to recover the velocity field.

Fluid Up-Sampling Methods One further approach, which is commonly used in Eulerian smoke simulations, is to use coarse grids for the simulation and to increase the resolution afterwards by using fine scale turbulence models [Kim et al. 2008; Schechter and Bridson 2008]. Similar approaches have been proposed to increase the resolution of Eulerian liquid simulations [Kim et al. 2013; Narain et al. 2008]. Also in the Lagrangian setting methods for increasing the resolution of the velocity field [Shao et al. 2015; Yuan et al. 2012], adding high frequency turbulent surface details [Mercier et al. 2015] and enriching fluid surfaces with wave details [Yang et al. 2016] have been presented.

These methods are complementary to our approach. They address the problem of too coarse samplings by generating additional small scale visual details. In contrast we address the problem of numerical dissipation and our method produces additional turbulent details on larger scales. Since the up-sampling methods act as a post processing step, they should also be applicable in combination with our approach.
Micropolar Fluids The model of micropolar fluids was introduced by Eringen [1966]. Since then it was successfully used in many practical applications. Examples are the flow of colloidal suspensions and polymeric fluids [Busuke and Tatsuo 1969], liquid crystals [Lee and Eringen 1971], human and animal blood [Ariman et al. 1974] and lubricants [Khonsari and Brewe 1989]. An introduction to the theory of micropolar fluids can be found in the textbooks of Lukaszewicz [1999] and Eringen [2001].

Our motivation for using the micropolar model to simulate classical fluids like water is, that microrotations are additional sources of vorticity and turbulence as pointed out by Eringen [2001]. This additional vorticity results in visual effects that are not captured in standard SPH simulations due to numerical dissipation.

\section{GOVERNING EQUATIONS AND MICROPOLAR MATERIAL MODEL}

The most prominent mathematical model describing the dynamics of Newtonian fluids is the Navier-Stokes model. The model can be derived from the conservation law of linear momentum and by presuming that the mechanical stress is composed of isotropic pressure and a diffusing viscous term. An important assumption of the model is that the infinitesimally small particles which compose a fluid continuum are not subject to rotational motion. This also implies that the law of angular momentum conservation is identically fulfilled if and only if the stress tensor is symmetric.

In this section we introduce the concept of micropolar fluids and present a material model that generalizes the Navier-Stokes equations for the simulation of incompressible inviscid turbulent flow. Following the definition of Lukaszewicz [1999], a micropolar fluid follows constitutive laws modeled using a generally non-symmetric stress tensor. Moreover, the definition includes that the fluid consists of rigid, spherical (and therefore rotationally invariant) particles. Based on the non-symmetric stress measures, the micropolar model additionally models rotating motions of the infinitesimal spherical particles using an angular velocity field. Due to the additional rotational degrees of freedom, the generation of vortices is facilitated and a wider range of potential dynamic effects are captured by the model.

Micropolar materials have been investigated for several decades in the field of mechanical engineering. However, the classical micropolar constitutive equations [Eringen 2001; Lukaszewicz 1999] model viscous flow. Moreover, the viscosity parameters are also used to couple the translational and the rotational motion in a dissipative way which is not desired for inviscid or nearly inviscid flow. In the following, we derive a modified micropolar material model that is more suitable for the simulation of incompressible inviscid turbulent fluids.

\subsection{Linear and Angular Momentum Conservation and Incompressibility}

In the following we derive the equations for linear and angular motion. Analogously to the Navier-Stokes model, a fluid in a threedimensional domain is described by a density field $\rho: \mathbb{R}^{3} \rightarrow \mathbb{R}$ and a velocity field $\mathbf{v}: \mathbb{R}^{3} \times[0, \infty) \rightarrow \mathbb{R}^{3}$ that maps a point $\mathbf{x}$ at time $t$ to its velocity vector. Moreover, the model is augmented by a microrotation field $\omega: \mathbb{R}^{3} \times[0, \infty) \rightarrow \mathbb{R}^{3}$ that maps a point $\mathbf{x}$ at 
time $t$ to its angular velocity. The laws for conservation of linear and angular momentum are then defined as

$$
\begin{aligned}
\rho \frac{D \mathbf{v}}{D t} & =\nabla \cdot \mathbf{T}+\mathbf{f} \\
\rho \Theta \frac{D \omega}{D t} & =\nabla \cdot \mathbf{C}+\mathbf{T}_{\times}+\tau
\end{aligned}
$$

with $\left[\mathrm{T}_{\times}\right]_{i}=\sum_{j} \sum_{k} \epsilon_{i j k} T_{j k}$ and where $\mathbf{T}, \mathbf{f}$ and $\epsilon_{i j k}$ denote the stress tensor, the specific external force and the Levi-Civita symbol, respectively. The model is further augmented by the scalar, isotropic microinertia coefficient $\Theta$, the specific external torque $\tau$, and the couple stress tensor $\mathrm{C}$ that models stresses resulting from angular motion. A physical interpretation for the microinertia coefficient is that each infinitesimal fluid particle has a certain inertial resistance against rotational accelerations. As discussed by Chen et al. [2010] the quantity can be measured experimentally using Lagrangian velocities of tracer particles. However, we decided to choose the microinertia coefficient by experimentally exploring the effects for several example values. We finally set $\Theta=2$ in all of our results based on our experiences. We would further like to mention that $\Theta$ is independent of the size of an SPH particle as the parameter occurs in the continuous equation before discretization.

Since we intend to model incompressible materials, the fluid's density $\rho$ must not change over time. Consequently, the incompressibility condition can be directly derived from the continuity equation resulting in

$$
\frac{D \rho}{D t}=-\rho \nabla \cdot \mathbf{v}=0
$$

This also implies that the velocity field $\mathbf{v}$ must be divergence-free, i.e. $\nabla \cdot \mathbf{v}=0$.

\subsection{Constitutive Model}

In order to derive the equations of motion, a suitable constitutive model for inviscid and low-viscous fluids must be found. Moreover, we aim to develop a constitutive model that generalizes the NavierStokes equations. We mathematically model the stress tensor $\mathrm{T}$ and the coupling tensor $\mathrm{C}$ as

$$
\begin{aligned}
& \mathbf{T}=-p \mathbf{I}+\mu \nabla \mathbf{v}^{T}-\mu_{t} \nabla \mathbf{v}+\left(\mu+\mu_{t}\right) \omega^{\times} \\
& \mathbf{C}=c \nabla \omega^{T},
\end{aligned}
$$

where $\left[\omega^{\times}\right]_{j k}=\sum_{i} \epsilon_{j i k} \omega_{i}$ and $p$ and I denote pressure and identity matrix, respectively. The pressure term builds the basis of material models for fluids and is responsible for counteracting compression. In the Navier-Stokes equation, viscosity is modeled using a symmetric second order tensor, i.e. $\mu\left(\nabla \mathbf{v}+\nabla \mathbf{v}^{T}\right)$. In contrast, we additively decouple the tensor representation and provide individual material parameters $\mu$ and $\mu_{t}$. In the following, we will refer to $\mu$ and $\mu_{t}$ as dynamic viscosity and transfer coefficient, respectively. In order to ensure consistency with the second law of thermodynamics the material parameters must satisfy $\mu \geq 0$ and $\mu_{t} \geq-\mu$. Consequently, we can guarantee that the terms will only dissipate but never produce energy. The last term, i.e. $\left(\mu+\mu_{t}\right) \omega^{\times}$, is responsible for the transfer from rotational into linear motions and vice versa. Furthermore, this term can be physically interpreted as a model for friction between the infinitesimal material particles in the fluid. Finally, we model the coupling tensor as diffusive term solely dependent on the microrotation gradient and the dynamic rotational viscosity parameter $c$.

\subsection{Equations of Motion}

As the conservation laws and constitutive equations are now established, we can finally derive the equations of motion that build the basis for the numerical fluid simulation. By plugging the constitutive equations (4) and (5) into the conservation laws (1) and (2) and by applying the incompressibility condition (3), we arrive at the following representation

$$
\begin{aligned}
\frac{D \mathbf{v}}{D t} & =-\frac{1}{\rho} \nabla p+v \Delta \mathbf{v}+\left(v+v_{t}\right) \nabla \times \omega+\frac{\mathbf{f}}{\rho} \\
\Theta \frac{D \boldsymbol{\omega}}{D t} & =\zeta \Delta \omega+\left(v+v_{t}\right)(\nabla \times \mathbf{v}-2 \omega)+\frac{\boldsymbol{\tau}}{\rho} .
\end{aligned}
$$

Here, $v=\frac{\mu}{\rho}, v_{t}=\frac{\mu_{t}}{\rho}$, and $\zeta=\frac{c}{\rho}$ denote the kinematic viscosity, the kinematic transfer coefficient and the kinematic rotational viscosity, respectively. Our formulation reduces to the classical Navier-Stokes equations when $v_{t}=-v, \zeta=0$, and $\tau=0$ which also proves that our model is a generalization of the Navier-Stokes model. It is also worth noting, that in our model the transfer between rotational and linear motion imposed by $v_{t}$ is, in contrast to $v$, non-dissipative since the coefficient is not involved in the diffusive terms.

Finally, for inviscid fluids the model can be reduced by choosing $v=0$ and $\zeta=0$ resulting in

$$
\begin{aligned}
\frac{D \mathbf{v}}{D t} & =-\frac{1}{\rho} \nabla p+v_{t} \nabla \times \omega+\frac{\mathbf{f}}{\rho} \\
\Theta \frac{D \boldsymbol{\omega}}{D t} & =v_{t}(\nabla \times \mathbf{v}-2 \omega)+\frac{\boldsymbol{\tau}}{\rho} .
\end{aligned}
$$

\section{SMOOTHED PARTICLE HYDRODYNAMICS DISCRETIZATION}

In this work we use the Smoothed Particle Hydrodynamics approach to spatially discretize the governing equations. To solve these equations we have to compute the density, the pressure term, the transfer terms and the viscosity terms (only if viscosity is required).

Using SPH the density $\rho_{i}$ at a position $\mathbf{x}_{i}$ is determined by

$$
\rho_{i}=\sum_{j} m_{j} W_{i j}
$$

where $m_{j}$ denotes the mass of the neighboring particles $j$ and $W_{i j}=$ $W\left(\mathbf{x}_{i}-\mathbf{x}_{j}, h\right)$ denotes a smoothing kernel with smoothing length $h$. In our simulations we use the cubic spline kernel [Monaghan 1992]. However, also other kernel functions can be used.

Since we want to simulate incompressible fluids, we compute the pressure forces using an implicit pressure solver. Our approach can be easily combined with any SPH pressure solver. We successfully tested our micropolar material model in combination with the current pressure solvers PBF [Macklin and Müller 2013], IISPH [Ihmsen et al. 2014a] and DFSPH [Bender and Koschier 2017]. However, for all our experiments we used DFSPH because of its good performance and its stability.

To compute the transfer terms we have to determine the curl of the linear and angular velocity field. In previous works often the differenced curl operator [Monaghan 1992] is used, e.g. to implement 
vorticity confinement [Macklin and Müller 2013] where energy is added to the system to amplify the existing vorticity and to counteract artificial damping. However, in our work we prefer to use the symmetric curl operator to obtain energy conservation [Price 2010]:

$$
(\nabla \times \mathbf{A})_{i}=-\rho_{i} \sum_{j} m_{j}\left(\frac{\mathbf{A}_{i}}{\rho_{i}^{2}}+\frac{\mathbf{A}_{j}}{\rho_{j}^{2}}\right) \times \nabla_{i} W_{i j} .
$$

In this work we are interested in inviscid fluids. However, if viscosity is required, the Laplacian in the viscosity terms in Equations (6) and (7) can be determined using the following SPH approximation [Ihmsen et al. 2014b; Monaghan 1992]:

$$
\Delta A_{i}=2 \sum_{j} \frac{m_{j}}{\rho_{j}} A_{i j} \frac{\mathbf{x}_{i j} \cdot \nabla W_{i j}}{\left\|\mathbf{x}_{i j}\right\|^{2}+0.01 h^{2}},
$$

where $\mathbf{x}_{i j}=\mathbf{x}_{i}-\mathbf{x}_{j}$.

\section{SMOOTHING THE VELOCITY FIELD}

In our work we aim to simulate turbulent inviscid fluids. Therefore, we set the viscosity parameters $v$ and $\zeta$ to zero and removed the corresponding terms in Equations (8) and (9). But note that our micropolar material model can also be used for viscous fluids. In this case the viscosity terms $v \Delta \mathbf{v}$ and $\zeta \Delta \omega$ can be computed using the SPH approximation of the Laplacian [Ihmsen et al. 2014b].

The simulation of inviscid fluids using SPH has the problem that particles can pass through each other which leads to unphysical results since the velocity field can be locally multi-valued [Monaghan 1989, 2002]. To solve this problem Monaghan [1989] proposed to smooth the velocity field so that near particles move with almost identical velocities. This method, also known as XSPH [Monaghan 1992], conserves linear and angular momentum. In previous works XSPH is sometimes used to simulate artificial viscosity (e.g. [Schechter and Bridson 2012]) since it smoothes out turbulent details in the velocity field which lets the fluid look viscous. However, note that XSPH is non-dissipative. In this work we use XSPH due to its property that it transfers the velocity of a particle to its neighbors in absence of viscosity and use a low coefficient since we do not want to loose details.

When smoothing the velocity field using XSPH, the smoothed velocity $\hat{\mathbf{v}}_{i}$ for a particle is determined by

$$
\hat{\mathbf{v}}_{i}=\mathbf{v}_{i}+\epsilon \sum_{j} \frac{m_{j}}{\rho_{j}}\left(\mathbf{v}_{j}-\mathbf{v}_{i}\right) W_{i j},
$$

where $0 \leq \epsilon<1$ is a tunable parameter. In contrast to classical Navier-Stokes fluids, we have a field of angular velocities in our micropolar fluid model. Since we also want to transfer the angular velocity of a particle to its neighbors, we propose to apply XSPH to the angular velocity field as:

$$
\hat{\boldsymbol{\omega}}_{i}=\omega_{i}+\eta \sum_{j} \frac{m_{j}}{\rho_{j}}\left(\omega_{j}-\omega_{i}\right) W_{i j} .
$$

Note that while the original XSPH is used to avoid that particles pass through each other, our XSPH variant for angular velocities has a different purpose. It is required to transfer the angular velocity of particles in order to get larger vortices in absence of viscosity.

\section{RESULTS AND DISCUSSION}

In the following we show results for our novel micropolar fluid model and comparisons with the classical Navier-Stokes model. To perform the simulations, we integrated our micropolar model in the open-source fluid library SPlisHSPlasH [Bender 2017b]. In all simulations we used the parallel neighborhood search algorithm introduced by Ihmsen et al. [2011], the boundary handling proposed by Akinci et al. [2012] and an adaptive time-stepping based on the CFL condition. Moreover, we employed the implicit pressure solver DFSPH [Bender and Koschier 2017] and enforced an average volume deviation of less than $0.01 \%$ and a divergence-error of less than $0.1 \%$. In our experiments we simulate inviscid fluids $(v=0, \zeta=0)$ and smooth the linear and angular velocity fields using XSPH as discussed in Section 5. We used a relatively small value of $\epsilon=0.002$ since our goal is to simulate almost inviscid turbulent fluids and with large values the fluid tends to look viscous (cf. Section 5). To get larger vortices in our results we set $\eta$ to 0.125 . As already pointed out in Section 3.1 we set $\Theta=2$ in all of our simulations.

In this section for better readability we use the abbreviation $\mathrm{CSPH}$ for the classical SPH method where we solve the classical Navier-Stokes equations using DFSPH in combination with XSPH. If we compute additional vorticity confinement forces according to [Macklin and Müller 2013], we abbreviate the method by VC$\mathrm{SPH}$. The simulation with our micropolar fluid model we denote by MPSPH.

Lid-Driven Cavity To evaluate our method, we chose the liddriven cavity scenario, which is a standard benchmark in computational fluid dynamics [Ghia et al. 1982]. It is a 2D problem consisting of a square cavity with edge lengths $L=1 \mathrm{~m}$, that is completely filled with fluid. The fluid is accelerated by a horizontally moving lid, such that the fluid flows with a velocity of $|\mathbf{v}|=1 \mathrm{~m} / \mathrm{s}$ in horizontal direction at the upper boundary. At the remaining boundaries, noslip conditions are applied. In our case these conditions are enforced by the method described in [Cummins and Rudman 1999]. For each fluid particle $i$ that is closer than the support radius to the boundary, a ghost particle is created by reflecting the particle at the boundary. Its velocity $\mathbf{v}_{i}^{g}$ is set to $\mathbf{v}_{i}^{g}=2 \mathbf{v}^{b}-\mathbf{v}_{i}$, such that the interpolated velocity at the boundary becomes $\mathbf{v}^{b}$. Further there is no gravity. The simulation was initialized with $12 \mathrm{k}$ fluid particles such that the that the cavity was completely filled. For the $2 \mathrm{D}$ simulations the 2D cubic spline kernel [Monaghan 1992] was used. The kinematic viscosity $v$ is set, such that we get a Reynolds number $R e=\frac{|\mathbf{v}| L}{v}$ of 10000, which was also used in [Ghia et al. 1982].

According to the results presented in [Ghia et al. 1982], which were achieved with a multi-grid finite-difference solver, we expect that this system develops a steady state flow. There should be one large vortex in the center and three smaller vortices in the corners of the cavity which rotate in the opposite direction as the central vortex. Figure 2 shows the resulting velocity fields of CSPH (left), VCSPH (middle) and our micropolar model (right). To obtain these velocity fields we simulated the scenario for a longer time period until we reached a steady flow. The classical SPH simulation shows the large central vortex, but does not produce the smaller vortices in the corners. We think the reason is the numerical diffusion of SPH which was already discussed in previous works [Cornelis et al. 2014; de Goes et al. 2015; Ihmsen et al. 2014b]. With additional vorticity 

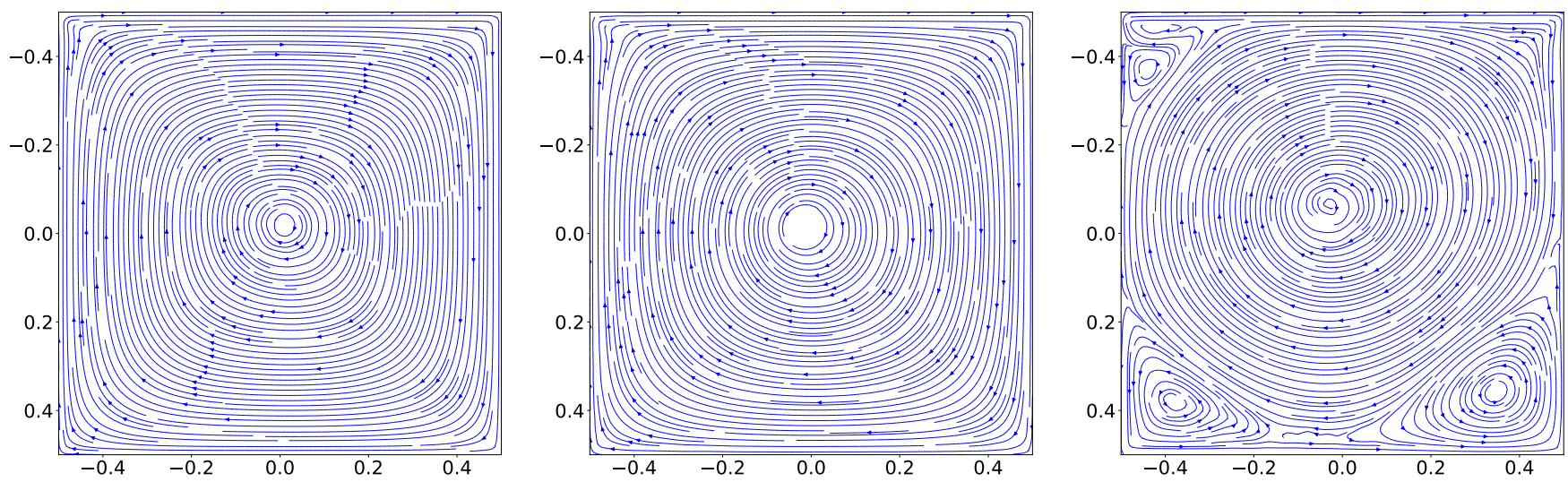

Figure 2: Velocity fields of the lid-driven cavity benchmark. CSPH (left) and VCSPH (middle) produce only one large central vortex. In contrast MPSPH (right) shows the expected results. It produces the central vortex and three smaller vortices in the corners which are rotating in the opposite direction.

confinement the picture looks similar since this method only amplifies the existing central vortex, but does not create the expected additional vortices in the corners. For this simulation we used the vorticity confinement approach of Macklin and Müller [2013] We tested various values of the vorticity confinement parameter $\varepsilon$ in their model, but only the rotation speed of the central vortex changed and no new vortices were formed. In Figure 2 the value was set to $\varepsilon=0.1$.

The micropolar SPH simulation shows the expected velocity profile with one additional vortex in the top left and two vortices in the bottom left and bottom right corners. In MPSPH the vorticity of the velocity field induces microrotations. The microrotations are advected along with the particle motion and are not much affected by numerical dissipation and the projection onto a divergence-free velocity field. These rotations are a source for new vortices. Further, as microrotations are transferred to nearby particles by XSPH, the formation of larger vortices is supported. An advantage of the micropolar model is that vorticity can be represented even in a single particle. VCSPH can represent vorticity only in particle neighborhoods. Moreover, in contrast to VCSPH micropolar fluids are linear and angular momentum conserving by construction. In summary it can be stated that the micropolar model is better suited for simulations of turbulent fluids than VCSPH because microrotations allow more complex interactions of particles than just accelerating existing vortices.

Comparisons To demonstrate the effect of the transfer coefficient $v_{t}$ in our micropolar model, we first simulated a dam break scenario with $1 \mathrm{M}$ fluid particles and a half sphere as static obstacle using increasing values of $v_{t}$ (see Figure 3 ). The simulation was initialized with a block of water on the left, which collapsed under gravity and flowed around the sphere. In the first simulation (top) we set $v_{t}=0$, which results in a classical SPH simulation without microrotations, and then increased $v_{t}$ from top to bottom. The comparison shows that turbulent details quickly get lost in the linear velocity field when using classical SPH. As already discussed for the lid-driven cavity example this happens due to numerical diffusion. With increasing the transfer coefficient $v_{t}$, we observe an

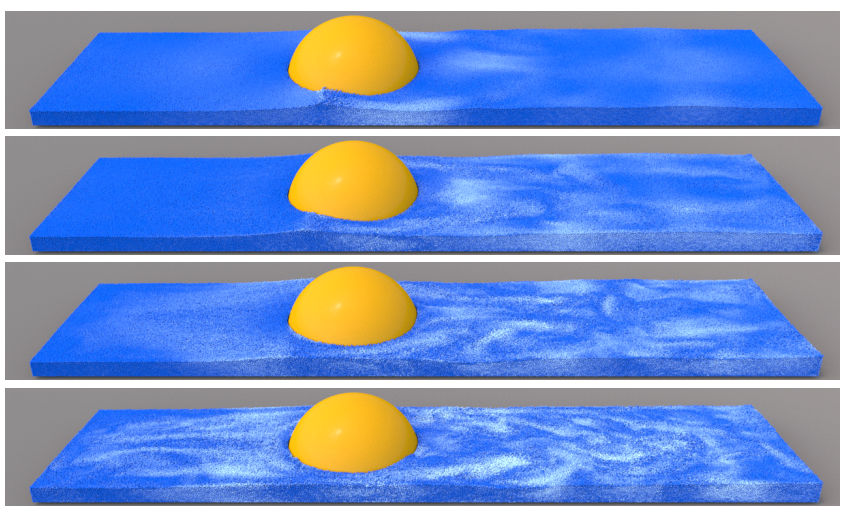

Figure 3: Breaking dam with $1 \mathrm{M}$ fluid particles and a static spherical obstacle simulated with four different transfer coefficients (from top to bottom): $v_{t}=0.0, v_{t}=0.05, v_{t}=0.075$ and $v_{t}=0.1$. The linear particle velocity is color coded, white refers to high and blue to low velocity.

increasing amount of turbulent details in the right part of the scene after the fluid flowed around the sphere. This demonstrates that our method allows for intuitive control of additional turbulence with a single parameter.

We also compared our approach with vorticity confinement using the same scenario. In Figure 4 a comparison of CSPH (top), VCSPH (middle) and MPSPH with $v_{t}=0.05$ (bottom) is depicted. Here we observe that artificially increasing the vorticity using the vorticity confinement method of Macklin and Müller adds energy to the system. This results in a faster fluid flow which can be seen in the middle image. In contrast to VCSPH our method treats energy in a physically consistent way and no additional energy is created. Moreover, in the end of the simulation in the accompanying video we can again observe that vorticity confinement only amplifies existing vortices while our micropolar model also supports the generations of new vortices. 

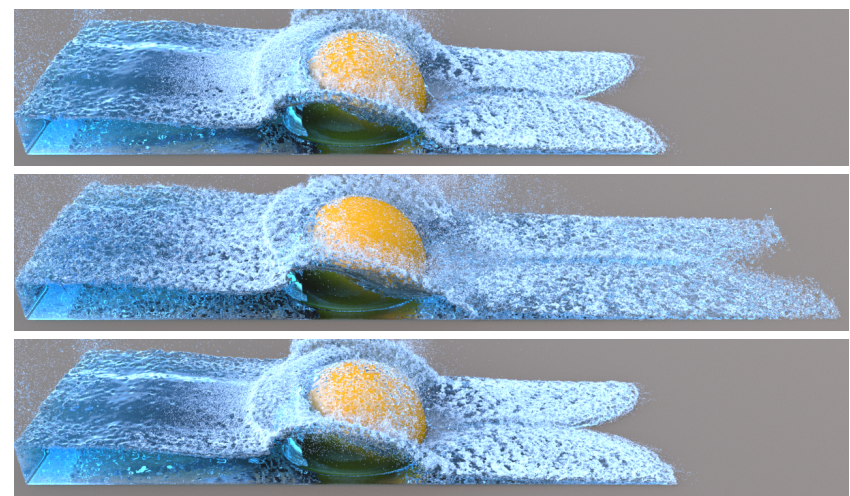

Figure 4: Comparison of CSPH (top), VCSPH (middle) and MPSPH with $v_{t}=0.05$ (bottom) in the dam break scenario with static spherical obstacle. Vorticity confinement adds energy to the fluid, which results in a much faster flow. In contrast MPSPH treats energy in a physically consistent way.

Stability Further, we simulated a more complex scene with dynamic boundary conditions and high particle velocities to show the stability and effect of our method (see Figure 1, right). On the left the fluid gets accelerated by a rotating propeller on the ground of the basin. The propeller rotates at 150 revolutions per minute. This results in high velocities of the fluid particles and a highly turbulent motion which is realistically handled by our approach The motion of the propeller was simulated using the implementation of position-based rigid body dynamics [Deul et al. 2014] in the open-source library PositionBasedDynamics [Bender 2017a].

In Figure 5 this scene is shown as particle view for CSPH and MPSPH with different values of the transfer coefficient $v_{t}$. The top image shows CSPH, where the turbulent details get lost due to numerical diffusion such that only few turbulences reach the right end of the scene. In the middle we see our MPSPH method with $v_{t}=0.2$ and on the bottom with $v_{t}=0.3$. The results show that increasing the transfer coefficient leads to more turbulent motion and the turbulences are transported farther to the right of the scene.

Performance In our last experiment we compared the computational effort of our method and CSPH in a breaking dam scenario with $1 \mathrm{M}$ fluid particles and three static Stanford dragons (see Figure 6). The times were measured on an Intel Xeon E5-2683 processor with $2.1 \mathrm{GHz}$ and 16 cores. The results show that most of the computation time in a simulation step is required for the neighborhood search and the implicit pressure solver. The additional computations for the microrotations in our micropolar fluid model have only a linear time complexity. In the dragon scenario this results in a negligible computational overhead of only $5 \%$ compared to the classical SPH approach. In larger scenarios as the river scene (see Figure 1, left) the computational overhead is even lower since the neighborhood search and the pressure solver require proportionally more time in more complex scenarios.

\section{CONCLUSION}

We presented an SPH based approach for simulating turbulent flows of incompressible inviscid fluids. Following a micropolar model the
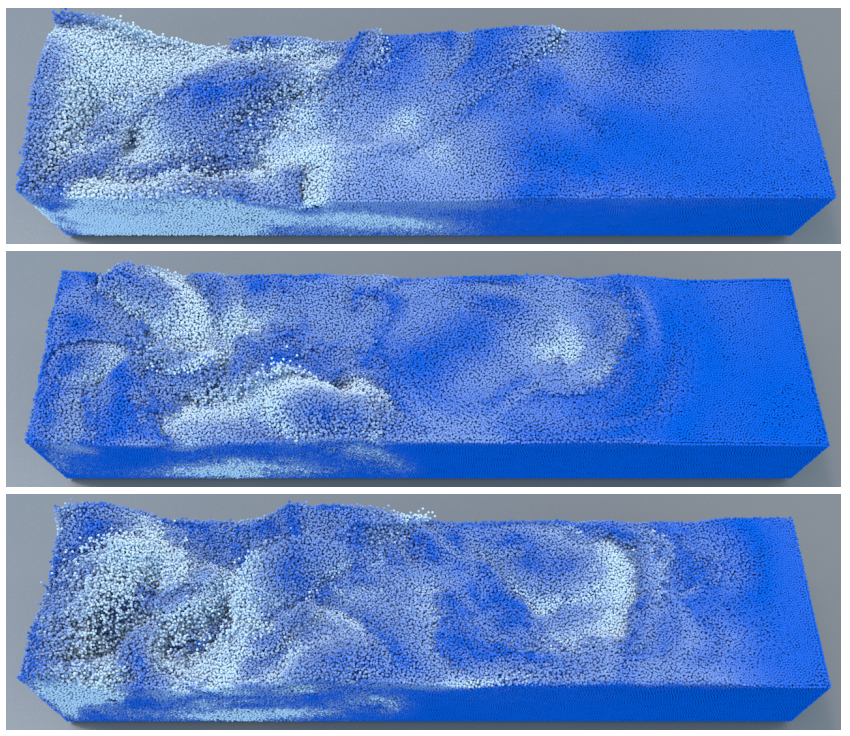

Figure 5: Propeller scene from Figure 1 rendered as particles with color coded linear velocities. The propeller in the left of the scene generates high fluid velocities and highly turbulent motion. Using CSPH (top) only a few turbulences reach the right part of the scene due to numerical diffusion. MPSPH with $v_{t}=0.2$ (middle) and with $v_{t}=0.3$ (bottom) results in much more turbulent motion and turbulences are transported farther to the right.

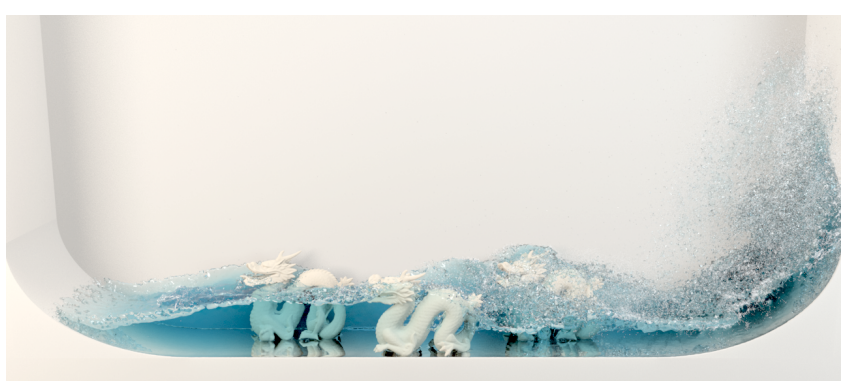

Figure 6: Breaking dam scenario with $1 \mathrm{M}$ fluid particles and three static obstacles.

field variables are augmented by a microrotation vector field that represents the angular velocity of infinitesimal particles in the fluid. We proposed a novel material model that generalizes the Navier-Stokes equations and facilitates the generation of vortices which results in highly turbulent flows with feature-rich surface details. The turbulence of the model can be controlled using a single parameter in the material model, i.e. the kinematic transfer coefficient $v_{t}$. In our results we demonstrated that the model produces physically meaningful results, generates interesting turbulent flows compared to the standard approach and robustly handles highly complex large-scale scenarios while the computational overhead is negligible. 
Due to the fact that our method is an SPH discretization of a generalization of the Navier-Stokes equations we could not make out any limitations beyond the already evident limitations of existing SPH based incompressible fluid solvers (see e.g. [Bender and Koschier 2017; Ihmsen et al. 2014a]).

In future work we would like to further investigate microstructural models for complex materials. Besides the micropolar approach that describes a material continuum with infinitesimal spherical particles subject to rotation, microstructural models also cover particles that are arbitrarily shaped or deforming. Moreover, we would like to investigate microstructural formulations for the simulation of elastic solids.

\section{ACKNOWLEDGMENTS}

We would like to thank Manuel Scholz for his kind support in providing the river model.

This work is supported by the German Research Foundation (DFG) under contract numbers BE 5132/3-1 and by the 'Excellence Initiative' of the German Federal and State Governments and the Graduate School of Computational Engineering at TU Darmstadt.

\section{REFERENCES}

N. Akinci, M. Ihmsen, G. Akinci, B. Solenthaler, and M. Teschner. 2012. Versatile rigid-fluid coupling for incompressible SPH. ACM Transactions on Graphics 31, 4 (2012), 62:1-62:8

A. Angelidis and F. Neyret. 2005. Simulation of smoke based on vortex filament primitives. In ACM SIGGRAPH / Eurographics Symposium on Computer Animation $87-96$.

T. Ariman, M. A. Turk, and N. D. Sylvester. 1974. Applications of microcontinuum fluid mechanics. International fournal of Engineering Science 12, 4 (1974), 273 - 293.

J. Bender. 2017a. PositionBasedDynamics Library.

https://github.com/InteractiveComputerGraphics/PositionBasedDynamics. (2017).

J. Bender. 2017b. SPlisHSPlasH Library.

https://github.com/InteractiveComputerGraphics/SPlisHSPlasH. (2017).

J. Bender and D. Koschier. 2017. Divergence-Free SPH for Incompressible and Viscous Fluids. IEEE Transactions on Visualization and Computer Graphics 23, 3 (2017), 1193-1206.

H. Busuke and T. Tatsuo. 1969. Two-dimensional shear flows of linear micropolar fluids. International fournal of Engineering Science 7, 5 (1969), 515-522.

J. Chen, C. Liang, and J. D. Lee. 2010. Theory and simulation of micropolar fluid dynamics. Proceedings of the Institution of Mechanical Engineers, Part N: Fournal of Nanoengineering and Nanosystems 224, 1-2 (2010), 31-39.

J. Cornelis, M. Ihmsen, A. Peer, and M. Teschner. 2014. IISPH-FLIP for Incompressible Fluids. Computer Graphics Forum 33, 2 (2014), 255-262.

S. J. Cummins and M. Rudman. 1999. An SPH Projection Method. f. Comput. Phys. 152 (1999), 584-607.

F. de Goes, C. Wallez, J. Huang, D. Pavlov, and M. Desbrun. 2015. Power Particles: An Incompressible Fluid Solver Based on Power Diagrams. ACM Transactions on Graphics 34, 4 (2015), 50:1-50:11.

C. Deul, P. Charrier, and J. Bender. 2014. Position-Based Rigid Body Dynamics. Computer Animation and Virtual Worlds 27, 2 (2014), 103-112.

A. C. Eringen. 1966. Theory of micropolar fluids. Fournal of Mathematics and Mechanics 16, No. 1 (1966), 1-16.

A. C. Eringen. 2001. Microcontinuum Field Theories: II. Fluent Media.

R. Fedkiw, J. Stam, and H. W. Jensen. 2001. Visual simulation of smoke. In Proceedings of the 28th annual conference on Computer graphics and interactive techniques. 15-22.

U. Ghia, K. N. Ghia, and C. T. Shin. 1982. High-Resolutions for incompressible flow using the Navier-Stokes equations and a multigrid method. F. Comput. Phys. 48, 3 (1982), $387-411$

A. Golas, R. Narain, J. Sewall, P. Krajcevski, P. Dubey, and M. Lin. 2012. Large-scale fluid simulation using velocity-vorticity domain decomposition. ACM Transactions on Graphics 31, 6 (2012), 148.

J.-M. Hong, H.-Y. Lee, J.-C. Yoon, and C.-H. Kim. 2008. Bubbles alive. In ACM Transactions on Graphics, Vol. 27. 48.

M. Ihmsen, N. Akinci, M. Becker, and M. Teschner. 2011. A Parallel SPH Implementation on Multi-Core CPUs. Computer Graphics Forum 30, 1 (2011), 99-112.

M. Ihmsen, J. Cornelis, B. Solenthaler, C. Horvath, and M. Teschner. 2014a. Implicit incompressible SPH. IEEE Transactions on Visualization and Computer Graphics 20 (2014), 426-435.
M. Ihmsen, J. Orthmann, B. Solenthaler, A. Kolb, and M. Teschner. 2014b. SPH Fluids in Computer Graphics. Eurographics (State of the Art Reports) (2014), 21-42.

T. Jang, H. Kim, J. Bae, J. Seo, and J. Noh. 2010. Multilevel Vorticity Confinement for Water Turbulence Simulation. The Visual Computer 26, 6-8 (2010), 873-881.

M. M. Khonsari and D. E. Brewe. 1989. On the Performance of Finite Journal Bearings Lubricated with Micropolar Fluids. Tribology Transactions 32, 2 (1989), 155-160.

T. Kim, J. Tessendorf, and N. Thürey. 2013. Closest point turbulence for liquid surfaces. ACM Transactions on Graphics 32, 2 (2013), 15.

T. Kim, N. Thürey, D. James, and M. Gross. 2008. Wavelet turbulence for fluid simulation. In ACM Transactions on Graphics, Vol. 27. 50.

J. D. Lee and A. C. Eringen. 1971. Boundary Effects of Orientation of Nematic Liquid Crystals. The fournal of Chemical Physics 55, 9 (1971), 4509-4512.

M. Lentine, M. Aanjaneya, and R. Fedkiw. 2011. Mass and Momentum Conservation for Fluid Simulation. In ACM SIGGRAPH / Eurographics Symposium on Computer Animation. 91-100.

G. Lukaszewicz. 1999. Micropolar Fluids: Theory and Applications. Birkhäuser.

M. Macklin and M. Müller. 2013. Position based fluids. ACM Transactions on Graphics 32, 4 (2013), 104

O. Mercier, C. Beauchemin, N. Thürey, T. Kim, and D. Nowrouzezahrai. 2015. Surface turbulence for particle-based liquid simulations. ACM Transactions on Graphics 34, 6 (2015), 202.

J. J. Monaghan. 1989. On the Problem of Penetration in Particle Methods. F. Comput. Phys. 82, 1 (1989), 1-15.

J. J. Monaghan. 1992. Smoothed particle hydrodynamics. Annual review of astronomy and astrophysics 30 (1992), 543-574.

J. J. Monaghan. 2002. SPH compressible turbulence. Monthly Notices of the Royal Astronomical Society 335, 3 (2002), 843-852.

R. Narain, J. Sewall, M. Carlson, and M. Lin. 2008. Fast animation of turbulence using energy transport and procedural synthesis. In ACM Transactions on Graphics, Vol. 27. 166.

S. I. Park and M. J. Kim. 2005. Vortex fluid for gaseous phenomena. In ACM SIGGRAPH / Eurographics Symposium on Computer Animation. 261-270.

T. Pfaff, N. Thürey, and M. Gross. 2012. Lagrangian vortex sheets for animating fluids. ACM Transactions on Graphics 31, 4 (2012), 112.

S. Premože, T. Tasdizen, J. Bigler, A. Lefohn, and R. T. Whitaker. 2003. Particle-Based Simulation of Fluids. Computer Graphics Forum 22, 3 (2003), 401-410.

D. J. Price. 2010. Smoothed Particle Magnetohydrodynamics - IV. Using the vector potential. Monthly Notices of the Royal Astronomical Society 401, 3 (2010), 1475

H. Schechter and R. Bridson. 2008. Evolving sub-grid turbulence for smoke animation. In ACM SIGGRAPH / Eurographics Symposium on Computer Animation. 1-7.

H. Schechter and R. Bridson. 2012. Ghost SPH for animating water. ACM Transactions on Graphics 31, 4 (2012), 61:1-61:8.

A. Selle, N. Rasmussen, and R. Fedkiw. 2005. A vortex particle method for smoke, water and explosions. In ACM Transactions on Graphics, Vol. 24. 910-914.

X. Shao, Z. Zhou, J. Zhang, and W. Wu. 2015. Realistic and stable simulation of turbulent details behind objects in smoothed-particle hydrodynamics fluids. Computer Animation and Virtual Worlds 26, 1 (2015), 79-94.

B. Solenthaler and R. Pajarola. 2009. Predictive-corrective incompressible SPH. In ACM Transactions on Graphics, Vol. 28. 40.

S. Weißmann and U. Pinkall. 2009. Real-time Interactive Simulation of Smoke Using Discrete Integrable Vortex Filaments. In Workshop in Virtual Reality Interactions and Physical Simulation "VRIPHYS" (2009).

S. Weißmann and U. Pinkall. 2010. Filament-based smoke with vortex shedding and variational reconnection. In ACM Transactions on Graphics, Vol. 29. 115

S. Yang, X. He, H. Wang, S. Li, G. Wang, E. Wu, and K. Zhou. 2016. Enriching SPH simulation by approximate capillary waves. In ACM SIGGRAPH / Eurographics Symposium on Computer Animation. 29-36.

Z. Yuan, Y. Zhao, and F. Chen. 2012. Incorporating stochastic turbulence in particlebased fluid simulation. The Visual Computer 28, 5 (2012), 435-444.

X. Zhang and R. Bridson. 2014. A PPPM fast summation method for fluids and beyond. ACM Transactions on Graphics 33, 6 (2014), 206.

X. Zhang, R. Bridson, and C. Greif. 2015. Restoring the missing vorticity in advectionprojection fluid solvers. ACM Transactions on Graphics 34, 4 (2015), 52.

B. Zhu, X. Yang, and Y. Fan. 2010. Creating and Preserving Vortical Details in SPH Fluid. Computer Graphics Forum 29, 7 (2010), 2207-2214. 